\title{
Source Index
}

(References are to first page of papers)

\begin{tabular}{|c|c|}
\hline $0016+73$ & 71 \\
\hline $0017+154$ & 65 \\
\hline $0055+300$ & 75 \\
\hline $0104+32$ & 75 \\
\hline $0106+131$ & 73 \\
\hline $0134+329$ & 115 \\
\hline $0138+136$ & 111 \\
\hline $0153+744$ & 7,71 \\
\hline $0212+735$ & $7,65,71,359,427$ \\
\hline $0220+43$ & 75 \\
\hline $0221+276$ & 111 \\
\hline $0222+36$ & 75 \\
\hline $0223+171$ & 191 \\
\hline $0229+345$ & 65 \\
\hline $0235+164$ & $7,143,167$ \\
\hline $0237-233$ & 139 \\
\hline $0248+430$ & 523 \\
\hline $0249+436$ & 523 \\
\hline $0258+35$ & 75 \\
\hline $0316+413$ & $\begin{array}{l}7,37,91,93,121,441 \\
479,481,491\end{array}$ \\
\hline $0320+053$ & 125 \\
\hline $0331+39$ & 75 \\
\hline $0333+321$ & $7,25,35$ \\
\hline $0336-019$ & 457 \\
\hline $0358+004$ & 125 \\
\hline $0404+76$ & 123 \\
\hline $0415+379$ & 103 \\
\hline $0418+000$ & 141 \\
\hline $0420-014$ & 457 \\
\hline $0428+205$ & 141 \\
\hline $0429+415$ & $111,115,119$ \\
\hline $0430+052$ & $\begin{array}{c}7,25,27,29,31,43,55 \\
121,143,297,509\end{array}$ \\
\hline $0433+296$ & 481 \\
\hline $0438-436$ & 139 \\
\hline $0454+844$ & 65,71 \\
\hline $0500+019$ & 141 \\
\hline $0511+380$ & 481 \\
\hline $0518+165$ & 111,125 \\
\hline $0521-365$ & 185 \\
\hline $0531+220$ & 481 \\
\hline $0537-441$ & 185 \\
\hline $0538+498$ & $7,95,129,509$ \\
\hline
\end{tabular}

\begin{tabular}{|c|c|}
\hline $0548+165$ & 125 \\
\hline $0552+398$ & $33,41,45$ \\
\hline $0605-085$ & 297 \\
\hline $0615+82$ & 71 \\
\hline $0710+439$ & 7,65 \\
\hline $0711+356$ & 7,141 \\
\hline $0716+71$ & 71 \\
\hline $0723+679$ & $7,25,65,99$ \\
\hline $0735+178$ & $7,117,143,167$ \\
\hline $0742+103$ & 141 \\
\hline $0742+318$ & 133 \\
\hline $0755+37$ & 75 \\
\hline $0809+483$ & 481 \\
\hline $0828+493$ & 141 \\
\hline $0831+557$ & 141 \\
\hline $0835+576$ & 135 \\
\hline $0836+29$ & 75 \\
\hline $0836+710$ & 71,165 \\
\hline $0838+133$ & 99 \\
\hline $0850+581$ & 7,133 \\
\hline $0851+202$ & $7,97,117,143,159$ \\
\hline $0855+143$ & 99 \\
\hline $0859+470$ & 141 \\
\hline $0906+431$ & $7,25,65$ \\
\hline $0923+392$ & $7,39,41,43,359,501$ \\
\hline $0945+408$ & 65 \\
\hline $0954+658$ & 299,301 \\
\hline $0957+561$ & $191,201,207$ \\
\hline $1003+350$ & 127,509 \\
\hline $1005+077$ & 111 \\
\hline $1037+30$ & 75 \\
\hline $1038+528$ & 101 \\
\hline $1039+81$ & 71 \\
\hline $1040+123$ & $7,25,99$ \\
\hline $1042+178$ & 191 \\
\hline $1055+201$ & 133 \\
\hline $1100+772$ & 99,509 \\
\hline $1117+14$ & 117,297 \\
\hline $1137+660$ & $7,25,99,133,519$ \\
\hline $1142+20$ & 75 \\
\hline $1144+35$ & 75 \\
\hline $1148-001$ & 121 \\
\hline $1150+49$ & 123 \\
\hline $1150+812$ & 7,71 \\
\hline
\end{tabular}




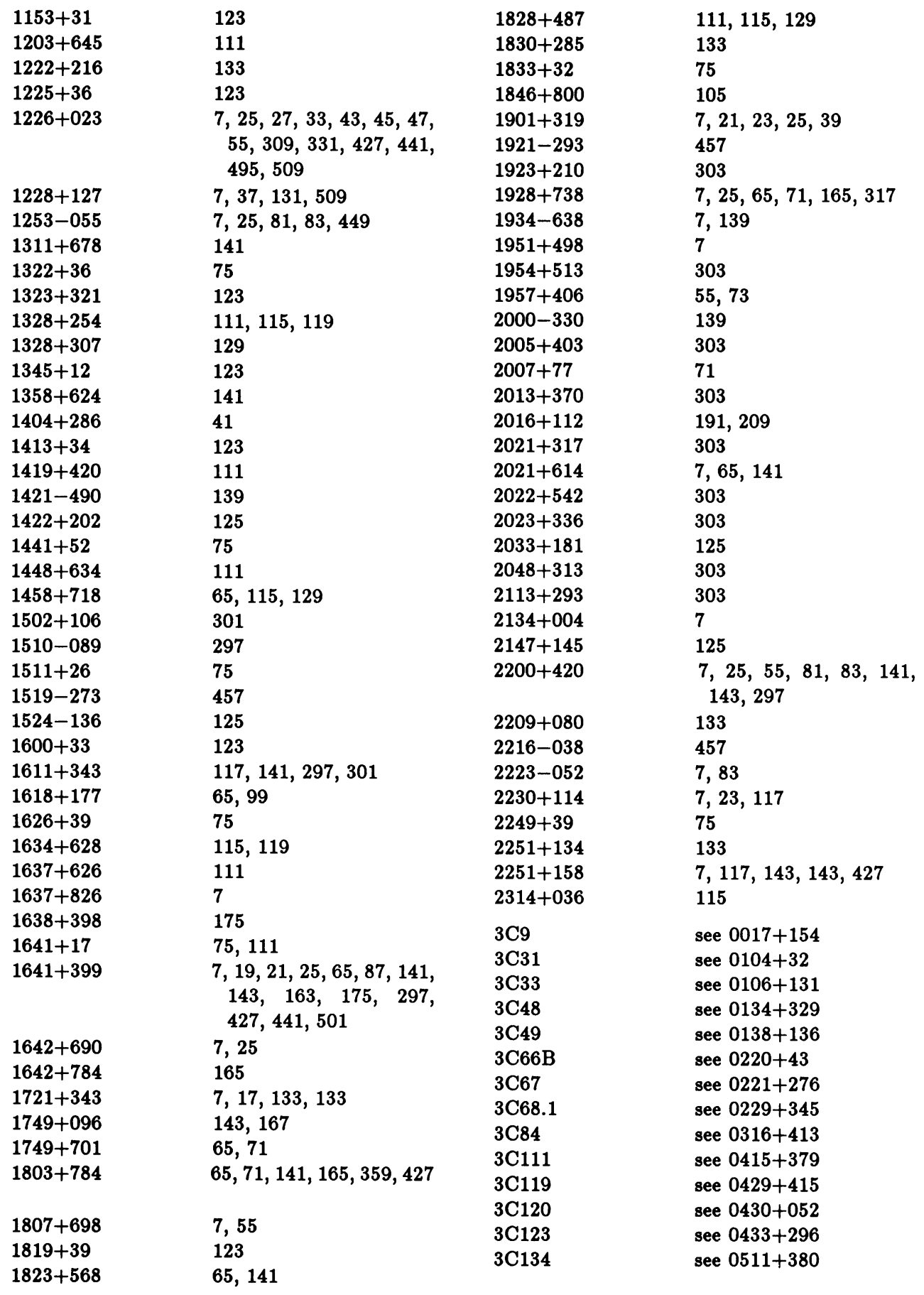




\begin{tabular}{|c|c|}
\hline $3 \mathrm{C138}$ & see $0518+165$ \\
\hline $3 \mathrm{C} 144$ & see $0531+220$ \\
\hline 3C147 & see $0538+498$ \\
\hline $3 \mathrm{C179}$ & see $0723+679$ \\
\hline 3C196 & see $0809+483$ \\
\hline $3 \mathrm{C} 205$ & see $0835+576$ \\
\hline $3 \mathrm{C} 207$ & see $0838+133$ \\
\hline $3 \mathrm{C} 212$ & see $0855+143$ \\
\hline $3 \mathrm{C} 216$ & see $0906+431$ \\
\hline $3 \mathrm{C} 236$ & see $1003+350$ \\
\hline $3 \mathrm{C} 237$ & see $1005+077$ \\
\hline $3 \mathrm{C} 245$ & see $1040+123$ \\
\hline $3 \mathrm{C} 249.1$ & see $1100+772$ \\
\hline $3 \mathrm{C} 263$ & see $1137+660$ \\
\hline $3 \mathrm{C} 264$ & see $1142+20$ \\
\hline $3 C 268.3$ & see $1203+645$ \\
\hline $3 \mathrm{C} 273$ & see $1226+023$ \\
\hline $3 \mathrm{C} 274$ & see $1228+127$ \\
\hline $3 \mathrm{C} 279$ & see $1253-055$ \\
\hline $3 \mathrm{C} 286$ & see $1328+307$ \\
\hline $3 \mathrm{C} 287$ & see $1328+254$ \\
\hline 3C299 & see $1419+420$ \\
\hline $3 \mathrm{C} 303$ & see $1441+52$ \\
\hline $3 \mathrm{C} 305$ & see $1448+634$ \\
\hline 3C309.1 & see $1458+718$ \\
\hline $3 C 315$ & see $1511+26$ \\
\hline $3 \mathrm{C} 334$ & see $1618+177$ \\
\hline 3C338 & see $1626+39$ \\
\hline $3 \mathrm{C} 343$ & see $1634+628$ \\
\hline 3C343.1 & see $1637+626$ \\
\hline $3 C 345$ & see $1641+399$ \\
\hline $3 \mathrm{C} 346$ & see $1641+17$ \\
\hline 3C371 & see $1807+698$ \\
\hline $3 C 380$ & see $1828+487$ \\
\hline $3 C 382$ & see $1833+32$ \\
\hline 3 C390.3 & see $1846+800$ \\
\hline 3 C395 & see $1901+319$ \\
\hline $3 C 405$ & see $1957+406$ \\
\hline $3 C 437.1$ & see $2147+145$ \\
\hline $3 \mathrm{C} 446$ & see $2223-052$ \\
\hline $3 \mathrm{C452}$ & see $2249+39$ \\
\hline $3 C 454.3$ & see $2251+158$ \\
\hline 3C459 & see $2314+036$ \\
\hline $3 \mathrm{C} 461$ & see $\operatorname{Cas} A$ \\
\hline $4 C 09.56$ & see $1749+096$ \\
\hline $4 C 21.50$ & 117 \\
\hline $4 C 32.44$ & see $1323+321$ \\
\hline 4C34.37 & 17 \\
\hline 4C34.47 & 25 \\
\hline 4C39.25 & see $0923+392$ \\
\hline
\end{tabular}

4C55.16

4C62.22

4C73.18

54 Cam

Algol

Arp 148

Arp 220

AR Lac

$\beta$ Lyr

$\beta$ Per

BL Lac

BLI.17

CTA26

CTA102

Cas A

Cen A

Cir X-1

Circinus

Crab

Cyg A

Cyg OB1

Cyg X-1

Cyg X-3

Cyg loop

DA193

DA251

DA267

DA406

DR21(OH)

G41.9+58

G45.1+0.1

G127.8-0.0

G340.8-0.1

Gal Cen

HD216489

HD26337

HD77137

HR1099

HR5110

HR8752

IC10

IC4553

II Peg

II ZW 96 see $0831+557$

see $1358+624$

see $1928+738$

329

$265,283,323,327$

213

261

$265,265,283$

329

329

see $2200+420$

see $0415+379$

see $0336-019$

see $2230+114$

$187,481,509$

137, 139, 441

139,265

213

see $0531+220$

see $1957+406$

303

265,327

$265,279,305$

287, 303

see $0552+398$

see $0831+557$

see $0923+392$

see $1611+343$

235

187

213, 263

213

251

see Sgr A*

329

265

265

$265,283,327$

265,327

329

213

213

265

213 


\begin{tabular}{|c|c|c|c|}
\hline III ZW 35 & 213 & ON1 & 235 \\
\hline IRAS1017+08 & 213 & OQ208 & see $1404+286$ \\
\hline IRAS1150-388 & 213 & OR103 & see $1502+106$ \\
\hline IRAS1211+03 & 213 & OT081 & 83 \\
\hline IRAS1639-096 & 213 & OW637 & see $2021+614$ \\
\hline IRAS1720-000 & 213 & OrionKL & $169,213,233,253,257$ \\
\hline IRC +10420 & 213 & & \\
\hline Jupiter & 309 & $\begin{array}{l}\text { P Cyg } \\
\text { Per A }\end{array}$ & $\begin{array}{l}329 \\
\text { see } 0316+413\end{array}$ \\
\hline LSI61303 & 265,327 & & \\
\hline M1 & see $0531+220$ & Fiuto & 309 \\
\hline M33 & 169 & R Cas & 259 \\
\hline M82 & 187 & RS CVn & 265 \\
\hline M87 & see $1228+127$ & RS Ophiuchi & 277 \\
\hline M100 & 175 & SK69202 & see Sanduleak star \\
\hline MW0309+41 & 323 & SN1970G & 187 \\
\hline Mars & 309 & SN1979C & 175,187 \\
\hline Mercury & 309 & SN1980K & 175,187 \\
\hline Moon & 309 & SN1981K & 187 \\
\hline Mrk231 & 213 & SN1986J & 175,187 \\
\hline Mrk273 & 213 & SN1987A & $185,189,487$ \\
\hline NGC315 & see $0055+300$ & $\begin{array}{l}\text { SS433 } \\
\text { S7. Pac }\end{array}$ & 265,275 \\
\hline NGC891 & 175,187 & SZ Psc & $265,283,327$ \\
\hline NGC1068 & $115,213,231$ & Sanduleak star & 189 \\
\hline NGC1167 & see $0258+35$ & Saturn & 309 \\
\hline NGC1275 & see $0316+413$ & $\sigma \mathrm{CrB}$ & 265,327 \\
\hline $\begin{array}{l}\text { NGC2484 } \\
\text { NGC3079 }\end{array}$ & $\begin{array}{l}\text { see } 0755+37 \\
169,213,231,233\end{array}$ & Sco X-1 & 265,281 \\
\hline NGC3690 & 213 & Sgr $A^{*}$ & $139,169,441$ \\
\hline NGC4258 & $169,213,231$ & Sgr B2 & $169,213,287,295$ \\
\hline $\begin{array}{l}\text { NGC4418 } \\
\text { NGC4449 }\end{array}$ & $\begin{array}{l}213 \\
187\end{array}$ & Sun & 309 \\
\hline NGC4486 & see $1228+127$ & Tau A & see $0531+220$ \\
\hline NGC4945 & 213 & U Ori & 213 \\
\hline NGC5141 & see $1322+36$ & UX Ari & $265,283,327$ \\
\hline NGC6251 & see $1637+826$ & Uranus & - \\
\hline NGC6334 & 263,287 & Uranus & 309 \\
\hline $\begin{array}{l}\text { NGC6946 } \\
\text { NGC7538 }\end{array}$ & $\begin{array}{l}175 \\
263,263\end{array}$ & VX Sgr & 259 \\
\hline NRAO140 & see $0333+321$ & Vela pulsar & 139 \\
\hline NRAO512 & see $1638+398$ & Venus & $309,319,479$ \\
\hline Neptune & 309 & Virgo A & see $1228+127$ \\
\hline OG003 & see $0500+019$ & W3 & 235 \\
\hline OI318 & see $0711+356$ & W3(OH) & $213,235,255$ \\
\hline OJ287 & see $0851+202$ & W43A & 249 \\
\hline OJ499 & see $0859+470$ & W49 & $169,223,233$ \\
\hline
\end{tabular}


W51

W75N

ZW1510+074
$169,169,213,223$

213

213
ZW475.056

ZZ Boo

213

329 